\title{
Creative use of novel glucose-lowering drugs for type 2 diabetes: where will we head in the next 50 years?
}

\author{
Bo Ahrén
}

Received: 28 January 2015 / Accepted: 16 February 2015 / Published online: 20 March 2015

(C) Springer-Verlag Berlin Heidelberg 2015

\begin{abstract}
Research conducted over the last 50 years has produced discoveries on the importance of glucose control for reducing the risk of diabetic complications, the pathophysiology of type 2 diabetes, the development and validation of mechanistic glucose-lowering targets, and the preclinical and clinical development of individual drugs. This science established the different drug classes that are clinically used today in association with lifestyle changes for lowering glucose in type 2 diabetes. For the next 50 years, we can anticipate that science will explore (1) the use of current drugs and, as they become available, newly developed drugs in early (initial) oral combinations followed by intensification with injectable combinations when glycaemia deteriorates, (2) the validation of novel mechanistic biochemical and physiological targets, including indirect effects of future antiobesity drugs, and (3) the development of true disease-modifying strategies based on knowledge of islet cell biology and replacement. This is one of a series of commentaries under the banner ' 50 years forward', giving personal opinions on future perspectives in diabetes, to celebrate the 50th anniversary of Diabetologia (1965-2015).
\end{abstract}

Keywords Combination therapy · DPP-4 inhibition · GLP-1 receptor agonists · Insulin · Metformin · SGLT-2 inhibition · Sulfonylurea $\cdot$ Thiazolidinediones $\cdot$ Type 2 diabetes
50 years of focus on glucose-lowering therapy: how have we arrived at where we are?

Over the last 50 years, several novel classes of glucoselowering drugs have been developed with different targets and mechanisms of action in type 2 diabetes. Four main scientific areas have paved the way for this development. First, it has been established that reducing hyperglycaemia prevents the onset and progression of secondary complications, and that it is important to start and intensify therapy as early as possible after deterioration of glycaemic control. Second, studies have established that islet dysfunction is the key pathophysiology of hyperglycaemia, but also that other factors, such as insulin resistance, defective incretin action and glucose toxicity, may contribute. Third, studies have identified several mechanistic cellular biological processes as potential targets for glucose-lowering therapy and have established that compounds that act at these targets may reduce circulating glucose. Fourth, interventional, preclinical and clinical studies have developed novel drugs and implemented them in the healthcare system. These four areas of research have been fundamental to our current understanding (Fig. 1). In fact, integrating the results of these four areas of research has been the driving force for development, as has crosstalk between academic and industrial research.

\section{Where are we now? Three concepts of today \\ Concept 1: Early and intensified glucose-lowering therapy is important}

Glucose lowering is important for the overall management of type 2 diabetes, although its intensification may need to be tempered in certain groups, such as in elderly and frail individuals and in those with cardiovascular diseases, to avoid side effects and risks, as discussed in the recent joint position 


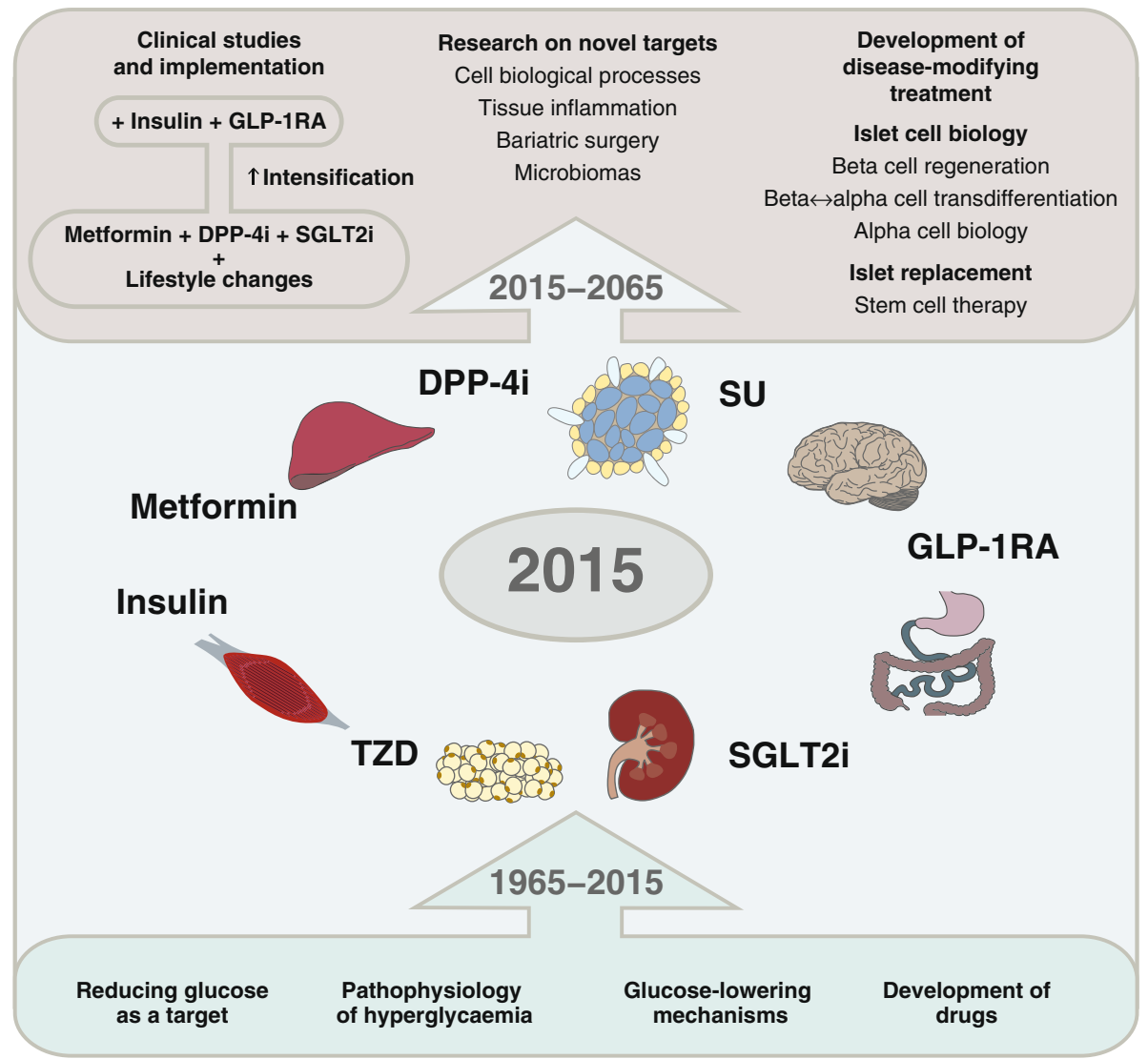

Fig. 1 We now have extensive knowledge on several glucose-lowering drugs in the healthcare system that can be combined with lifestyle modifications, namely, metformin, sulfonylureas (SU), DPP-4 inhibitors (DPP-4i), GLP-1 receptor agonists (GLP-1RA), SGLT2 inhibitors (SGLT2i), TZDs and insulin. These drugs target different processes involved in glucose control, as indicated in the middle panel. This knowledge is based on fundamental research carried out over the last 50 years, as depicted in the bottom panel. Over the next 50 years we can expect the

document from the EASD and the ADA [1]. A strong rationale for initiating glucose-lowering therapy early after diagnosis and intensifying treatment early after worsening of glycaemic control has also been provided by findings that the time spent in hyperglycaemia is a major contributor to microvascular complications and also significantly contributes to macrovascular complications [2]. Consequently, preventing hyperglycaemia by intensified glucose-lowering reduces the risk for and progression of these complications. Early therapy and earlier diagnosis have also resulted in reduced overall mortality in diabetes. It is therefore important to target glycaemia during the early stages of the disease and early after progression of the disease.

Concept 2: Treatment should target physiological processes

To affect circulating glucose levels, it is important to target the mechanistic processes involved in controlling glucose concentrations. Islet dysfunction is the key factor underlying the use of these strategies in an algorithm with early combination of oral agents together with lifestyle changes (metformin+DPP-4i+SGLT2i) followed by intensification with injectables in combination (insulin+ GLP-1RA), along with research aimed at developing new targets and, most importantly, identifying true disease-modifying strategies based on increased knowledge of islet cell biology and development of islet cell replacement

development of hyperglycaemia and the progressive glycaemic deterioration associated with type 2 diabetes, but other processes are also involved in the regulation of glucose homeostasis [3]. These processes are all potential targets for therapy and fundamental both for the effects of lifestyle changes [4] and for the effects of several classes of therapeutic agents. Some targets to be used as glucose-lowering therapy have been recommended in the joint EASD/ADA 2015 position statement update [1].

Islet dysfunction Islet dysfunction is the key defect in type 2 diabetes and is characterised by impaired insulin secretion, augmented glucagon secretion and reduced beta cell mass. Lifestyle changes, including energy intake restriction and body weight reduction, can produce improvements, which may be due to a reduction in ectopic fat infiltration [4]. Islet dysfunction is also targeted by drugs. The first drug class to target islet dysfunction was the sulfonylureas, which were introduced more than 50 years ago. They stimulate insulin 
secretion through a direct glucose-independent effect on beta cells [5]. Also glucagon-like peptide-1 (GLP-1) receptor agonists and dipeptidyl peptidase-4 (DPP-4) inhibitors, which were introduced during the 2000s, target islet dysfunction since they stimulate insulin secretion and inhibit glucagon secretion in a glucose-dependent manner [5].

Insulin resistance In addition to lifestyle changes [4], insulin resistance in the liver is targeted by metformin, which has been widely used as a glucose-lowering therapy for more than 50 years and is recommended as the first-line pharmacological therapy in most guidelines. Although its detailed mechanism of action still remains to be established, it reduces hepatic glucose output by inhibiting gluconeogenesis and increasing insulin-induced suppression of hepatic glucose production [5]. Developed during the 1990s, thiazolidinediones (TZDs) target insulin resistance in adipocytes. TZDs activate the transcription factor peroxisome proliferator-activated receptor $\gamma$ (PPAR $\gamma$ ), a key regulator of adipocyte development and function, resulting in adipogenesis, reduced release of NEFA and improved insulin sensitivity, although other mechanisms are also of relevance [5].

Other targets The stomach influences postprandial glucose levels since it delivers ingested food to the gut for further absorption, and gastric emptying can be delayed by GLP-1 receptor agonists. The kidney is also important. It has been known for several decades that prevention of glucose reabsorption in the proximal nephrons through inhibition of sodium-glucose co-transporter 2 (SGLT2) reduces glucose levels, and SGLT2 inhibitors have been introduced into the clinic in the last 3 years [6]. As central satiety mechanisms are involved in the regulation of body weight, the brain is another drug target, and GLP-1 receptor agonists exert an effect here. Stimulation of glucose uptake and utilisation in muscle and adipose tissue by exogenous insulin administration is a powerful glucose-lowering mechanism as well.

\section{Concept 3: Drugs should have a low risk of adverse events}

Besides having glucose-lowering abilities, it is important that novel drugs minimise the risk of hypoglycaemia, weight gain and other adverse events - a low risk of hypoglycaemia being particularly important [7]. The risk of hypoglycaemia is higher for sulfonylurea and exogenous insulin and lower for DPP-4 inhibitors, GLP-1 receptor agonists and SGLT2 inhibitors. Furthermore, those compounds associated with a greater risk, such as sulfonylurea and insulin, commonly result in weight gain because of eating in self-defence against hypoglycaemia. In contrast, weight neutrality is seen after DPP-4 inhibition, and varying degrees of weight reduction are seen after metformin, GLP-1 receptor agonists and SGLT2 inhibitors. Other described adverse events include oedema and increased risk of fractures with TZDs, and genital infections and volume depletion with SGLT2 inhibitors. At present, several very large outcomes trials are ongoing for newer compounds, with particular focus on cardiovascular safety [8].

\section{Challenges for the next 50 years: where will we go from here?}

One of the current challenges is that, despite lifestyle changes and the use of glucose-lowering drugs, glycaemic control is insufficient in many patients. There are many potential explanations for this, ranging from economic restraints over organisational aspects of the healthcare system, to poor compliance and insufficient potency of individual strategies. An important reason for poor glycaemic control is, however, clinical inertia, i.e. the delay in the initiation or intensification of glucose-lowering therapy. This may be due to the attitudes and/or level of knowledge of patients and healthcare providers, by the healthcare organisation and resources of the healthcare system or by fear of adverse events such as hypoglycaemia or weight gain when using glucose-lowering agents. However, together with lifestyle modifications, the glucose-lowering drugs of today have given us the tools to meet these challenges, although there is a need for further research to develop better glucose-lowering strategies to prevent clinical inertia and allow more efficient glucose control. Most guideline algorithms of today use lifestyle modifications as the basis and then stepwise intensification of glucose control: starting with metformin monotherapy, then adding a second drug, then a third drug and then complex insulin regimens ( \pm GLP-1 receptor agonists) at times of insufficient glucose control. However, the use of the presently available drugs can be further creatively optimised, and this will occur over the coming years. This means greater use of glucose-lowering drugs in combination to allow synergistic mechanisms. It also means that it is important to carry out studies to provide more information on treatment individualisation, to explore what drug works better in a particular group of patients, and to conduct studies to take account of patients' views about side effects.

\section{Optimising the use of the available glucose-lowering tools}

There is a need to optimise the use of the available drugs by using them in combination on a background of lifestyle modifications. By doing so, the philosophy of stepwise initiation of drug therapy will be replaced with a philosophy of initiating early combination therapy and using combinations when intensifying therapy. The healthcare provider may thus combine compounds with different mechanisms of action, and recent studies have documented optimised efficacy by combining 
agents [9]. In the early stages of the disease, combining lifestyle modifications with metformin or TZDs together with sulfonylurea or incretin therapy, or combining metformin with insulin are potential options. Metformin in combination with DPP-4 inhibition has the advantage of targeting insulin resistance and both the beta and alpha cell components of islet dysfunction, therefore offering a pathophysiological approach. In addition, it has been shown that metformin increases circulating levels of GLP-1, a mechanism that is augmented by the prevention of GLP-1 inactivation by DPP-4 inhibition. Thus, several mechanisms contribute to the synergistic effects of the combination of metformin plus a DPP-4 inhibitor, and this combination has indeed been shown to be effective at reducing glucose with a low risk of hypoglycaemia or other adverse events and no weight gain [10]. However, $\mathrm{HbA}_{1 \mathrm{c}}$ is not always normalised by this combination and often hits a floor of approximately 7\% [11]. Therefore, additional drugs may be introduced for a more complete normalisation of glycaemia. Ideally, such an added drug should work through another target, which would make the recently developed SGLT2 inhibitors a suitable choice; they prevent reabsorption of glucose in the kidney, which lowers circulating glucose [6]. Indeed, the addition of SGLT2 inhibition to metformin therapy improves glucose lowering with a low risk for hypoglycaemia and weight reduction. However, islet dysfunction is not targeted by this combination, therefore, metformin +DPP-4 inhibition+SGLT2-inhibition together with lifestyle modification may be the optimal early treatment, as this would mean that the liver, the islets, the gut and the kidney would be targeted by different mechanisms. Together, these therapies have the potential to strongly reduce circulating glucose with a low risk for hypoglycaemia and no weight gain. Now, basic, preclinical and clinical studies with this triple drug combination are warranted to further understand the potential mechanisms. The cost issue also needs to be addressed, since in many economies the current cost of these therapies prohibits their widespread use at present. Health economy analyses of various treatment options are therefore also warranted to assure a cost-benefit use of future therapy.

Due to the progressive nature of the disease, aggressive and early treatment with lifestyle modification in combination with metformin+DPP-4 inhibition+SGLT2 inhibition may eventually become insufficient for glycaemic control. It is then important to react early with the next step of glucose lowering therapy. The injectables-GLP-1 receptor agonists and insulin - have additive mechanisms with potential synergistic actions and may then be used [12]. The combination of GLP-1 receptor agonists and insulin has also been demonstrated to result in powerful reductions in $\mathrm{HbA}_{1 \mathrm{c}}$ with a low risk of hypoglycaemia and no weight gain. We will therefore have two potent drug combinations for glucose-lowering in T2D: one oral combination (metformin+DPP-4 inhibition+SGLT2 inhibition) and one injectable combination (insulin+GLP-1 receptor agonism). These two combinations thus may offer lasting glycaemic control, and studies and implementation of this strategy, as illustrated in Fig. 1, are warranted.

\section{Development of novel strategies}

The use of novel combinations of currently available drugs should not stop further research aiming to develop novel glucose-lowering strategies. This may be performed along the two lines discussed below.

\section{Development of novel targets}

Besides optimising the already available tools, novel targets to improve altered biochemical processes in type 2 diabetes are important. Potential targets include conventional cell biological signalling processes, a number of which are currently being investigated by promising ongoing studies, such as inhibition of $11 \beta$-hydroxysteroid dehydrogenase 1 , glucokinase activation, G protein-coupled fatty acid receptor activation, glucagon receptor antagonism and metabolic inhibition of hepatic glucose output [4]. Targets related to tissue inflammation and bariatric surgery need to be studied, and the importance of the microbiome also warrants further investigation.

\section{Development of true disease-modifying therapy}

Besides establishing combinations of existing drugs and deepening the understanding of the potential of known metabolic targets, it is of importance to concentrate research on finding true disease-modifying approaches. Such approaches need to concentrate on beta cell differentiation and regeneration, beta $\leftrightarrow$ alpha cell transdifferentiation, alpha cell biology and the development of stem cell-derived beta cell replacement [13].

\section{Conclusions and entering the avenue for the next 50 years}

In conclusion, research during the last 50 years has produced tools for lowering circulating glucose via different mechanisms and with a low risk of adverse events. It is now important to make use of the extensive knowledge and the tools we have to take appropriate steps in the coming 50 years. Basic research will generate knowledge on the combination therapy as presented here in more detail and clinical studies aiming at optimising implementing the two combination phases to replace the stepwise intensification paradigm with a combination paradigm will also be important. Future research should therefore be directed towards examining these combinations and their potential synergistic mechanisms. It should also aim to identify biomarkers that signal when combination therapy 
should be started or intensified, and to identify the characteristics of different patient groups to allow a more individualised treatment strategy. Research for target-finding is also important, including the development of true disease-modifying compounds, such as the prevention of beta cell degeneration or provision of novel stem cells to replace the diseased islet cells.

Acknowledgements The author thanks Linda Ahlkvist (Lund University, Lund, Sweden) for discussions related to Fig. 1.

Contribution statement The author was the sole contributor to this article.

Duality of interest The author has consulted for Novartis, GlaxoSmithKline, Merck, Sanofi, Novo Nordisk, Boehringer Ingelheim and Takeda, and has received lecture fees from Novartis, Merck, Novo Nordisk, Sanofi, Bristol Myers Squibb, AstraZeneca and GlaxoSmithKline, which all are companies that manufacture DPP-4 inhibitors or GLP-1 receptor agonists.

\section{References}

1. Inzucchi SE, Bergenstal RM, Buse JB et al (2015) Management of hyperglycaemia in type 2 diabetes. 2015: a patient-centred approach: update to a position statement of the American Diabetes Association and the European Association for the study of diabetes. Diabetologia $58: 429-442$
2. Holman RR, Paul SK, Bethel MA, Matthews DR, Neil HA (2008) 10-year follow-up of intensive glucose control in type 2 diabetes. N Engl J Med 359:1577-1589

3. Kahn SE, Cooper ME, del Prato S (2014) Pathophysiology and treatment of type 2 diabetes: perspectives on the past, present, and future. Lancet 383:1068-1083

4. Lim EL, Hollingsworth KG, Aribisala BS, Chen MJ, Mathers JC, Taylor R (2011) Reversal of type 2 diabetes: normalisation of beta cell function in association with decreased pancreas and liver triacylglycerol. Diabetologia 54:2506-2514

5. Tahrani AA, Bailey C, del Prato S, Barnett AH (2014) Management of type 2 diabetes: new and future developments in treatment. Lancet 378:182-197

6. Rosenwasser RF, Sultan S, Sutton D, Choksi R, Epstein BJ (2013) SGLT-2 inhibitors and their potential in the treatment of diabetes. Diabetes Metab Syndr Obes 6:453-467

7. Ahrén B (2013) Avoiding hypoglycemia: a key to success for glucoselowering therapy in type 2 diabetes. Vasc Health Risk Manag 9:155-163

8. Petrie JR (2013) The cardiovascular safety of incretin-based therapies: a review of the evidence. Cardiovasc Diabetol 12:130

9. Zinman B (2011) Initial combination therapy for type 2 diabetes mellitus: is it ready for prime time? Am J Med 124(Suppl 1):S19-S34

10. Ahrén B (2008) Novel combination treatment of type 2 diabetes. DPP-4 inhibition+metformin. Vasc Health Risk Manag 4:383-394

11. Deacon CF, Mannucci E, Ahrén B (2012) Glycemic efficacy of GLP-1 receptor agonists and DPP-4 inhibitors as add-on therapy to metformin in subjects with type 2 diabetes - a review and meta analysis. Diabetes Obes Metab 14:762-767

12. Ahrén B (2014) Insulin plus incretin. A glucose-lowering strategy for type 2-diabetes. World J Diabetes 15:40-51

13. Halban PA, Polonsky KS, Bowden DW et al (2014) $\beta$-Cell failure in type 2 diabetes: postulated mechanisms and prospects for prevention and treatment. J Clin Endocrinol Metab 99:1983-1992 